\title{
University of Groningen
}

\section{Modification of graphite surfaces for the adsorption of molecular motors}

\author{
Heideman, Henrieke
}

DOI:

10.33612/diss. 100690963

IMPORTANT NOTE: You are advised to consult the publisher's version (publisher's PDF) if you wish to cite from it. Please check the document version below.

Document Version

Publisher's PDF, also known as Version of record

Publication date:

2019

Link to publication in University of Groningen/UMCG research database

Citation for published version (APA):

Heideman, H. (2019). Modification of graphite surfaces for the adsorption of molecular motors. [Thesis fully internal (DIV), University of Groningen]. Rijksuniversiteit Groningen.

https://doi.org/10.33612/diss.100690963

\begin{abstract}
Copyright
Other than for strictly personal use, it is not permitted to download or to forward/distribute the text or part of it without the consent of the author(s) and/or copyright holder(s), unless the work is under an open content license (like Creative Commons).

The publication may also be distributed here under the terms of Article $25 f a$ of the Dutch Copyright Act, indicated by the "Taverne" license. More information can be found on the University of Groningen website: https://www.rug.nl/library/open-access/self-archiving-pure/taverne-
\end{abstract} amendment.

Take-down policy

If you believe that this document breaches copyright please contact us providing details, and we will remove access to the work immediately and investigate your claim.

Downloaded from the University of Groningen/UMCG research database (Pure): http://www.rug.nl/research/portal. For technical reasons the number of authors shown on this cover page is limited to 10 maximum. 


\section{Modification of Graphite Surfaces for the Adsorption of Molecular Motors}




\section{Wrijksuniversiteit groningen}

The work described in this thesis was carried out at the Stratingh Institute for Chemistry, University of Groningen, The Netherlands.

This work was financially supported by the Ministry of Education, Culture and Science (Gravitation program 024.001.035).

Cover design by Mathijs Mabesoone

Print: Ipskamp Printing, Enschede, The Netherlands

ISBN: 978-94-034-2132-2 (Printed Version)

ISBN: 978-94-034-2131-5 (Electronic Version) 


\title{
rijksuniversiteit groningen
}

\section{Modification of Graphite Surfaces for the Adsorption of Molecular Motors}

\author{
Proefschrift \\ ter verkrijging van de graad van doctor aan de \\ Rijksuniversiteit Groningen \\ op gezag van de \\ rector magnificus prof. dr. C. Wijmenga \\ en volgens besluit van het College voor Promoties.
}

De openbare verdediging zal plaatsvinden op

vrijdag 8 november om 16:15 uur

door

\section{Gerjanne Henrieke Heideman}

geboren op 10 maart 1991

te Zwolle 


\section{Promotores}

Prof. dr. B.L. Feringa

Prof. dr. M.A. Stöhr

\section{Beoordelingscommissie}

Prof. dr. K.-H. Ernst

Prof. dr. E. Otten

Prof. dr. J. G. Roelfes 


\section{Table of Contents}

Chapter 1: Introduction: Artificial Nanovehicles on Surfaces

1.1 Molecular motion on surfaces 10

1.2 Molecular designs of nanovehicles 11

1.3 Molecular motors based on overcrowded alkenes 13

1.4 Motorized nanovehicles 16

1.5 Aim of this research and thesis outline 19

1.6 References 20

Chapter 2: Scanning Tunneling Microscopy 25

2.1 Quantum tunneling 26

2.2 STM in practice 28

2.2.1 STM at the solid/liquid interface $\quad 29$

2.2.2 Sample preparation 29

2.3 References 30

Chapter 3: The Paramount Role of Internal Double Bonds in Discrete long Alkylated Naphthalenediimides Surface-Infrastructures $\quad 31$

3.1 Introduction 32

3.2 Discrete long alkylated naphthalenediimides $\quad 32$

3.3 Results and Discussion 33

3.3.1 Synthesis of alkylated naphthalenediimides 33

3.3.2 Self-assembly saturated NDIs 34

3.3.3 Self-assembly unsaturated NDIs 35

3.3.4 Pivotal role of the internal double bonds in the 2D-crystallization 37

3.3.5 Computational support of the experimental results 39

$\begin{array}{lll}3.4 & \text { Conclusion } & 41\end{array}$

3.5 Experimental 41

3.6 References 50 
Chapter 4: The Influence of Multiple Unsaturations in the Alkyl Chains of Naphthalenediimides on the Self-Assembly

4.1 Introduction 52

4.2 Discrete long alkylated naphthalenediimides $\quad 52$

4.3 Results and Discussion 53

4.3.1 Synthesis of alkylated naphthalenediimides 53

4.3.2 Self-assembly of $\left(\mathrm{u}_{2}\right) \mathrm{Cn}-\mathrm{NDI}-\left(\mathrm{u}_{2}\right) \mathrm{Cn} \quad 54$

4.3.3 Stereoisomer selectivity on the surface 57

4.3.4 Self-assembly of $\left(\mathrm{u}_{3}\right) \mathrm{Cn}$-NDI- $\left(\mathrm{u}_{3}\right) \mathrm{Cn} \quad 57$

4.3.5 Influence of the internal double bonds 59

4.4 Conclusion 61

4.5 Experimental 68

Chapter 5: Templated Alkoxy-Pyrene Adsorption within an Alkylated Naphthalenediimide Adlayer on Graphite $\quad 69$

$\begin{array}{lll}\text { 5.1 } & \text { Introduction } & 70 \\ 5.2 & \text { Results and discussion } & 71 \\ 5.2 .1 & \text { Alkoxy-pyrene adsorption } & 71 \\ 5.2 .2 & \text { Influence of solvent molecules } & 73 \\ 5.2 .3 & \text { Size dependence of alkoxy-pyrenes } & 73 \\ 5.2 .4 & \text { NDI core separation } & 74 \\ 5.2 .5 & \text { Adsorption motif } & 75 \\ 5.2 .6 & \text { Third-generation molecular motor } & 76 \\ 5.3 & \text { Conclusion } & 77 \\ 5.4 & \text { Experimental } & 77 \\ 5.5 & \text { References } & 85\end{array}$

Chapter 6: Surface Assembled Molecular Motors with Pyridine Moieties

$\begin{array}{lll}6.1 & \text { Introduction } & 90\end{array}$

6.2 Results and Discussion 92

6.2.1 ISA-O-C18 adlayer in 1-phenyloctane 92

6.2.2 Nanocorrals 95

6.2.3 ISA-0-C18 adlayer in different solvents 97

6.3 Conclusion 102

6.4 Experimental 102

6.5 References 105 
Chapter 7: Tailoring Third-Generation Molecular Motors for Surface Adsorption

$\begin{array}{lll}7.1 & \text { Introduction } & 110\end{array}$

$\begin{array}{lll}7.2 & \text { Results and discussion } & 110\end{array}$

$\begin{array}{lll}7.2 .1 & n \text {-pentacontane adlayer } & 110\end{array}$

7.2.2 Third-generation molecular motors on $n$-pentacontane adlayer 111

7.2.3 Synthesis third-generation molecular motors with long alkyl tails 113

7.2.4 Molecular motors with long alkyl tails on $n$-pentacontane adlayer 115

$\begin{array}{lll}7.3 \text { Conclusion } & 116\end{array}$

$\begin{array}{lll}7.4 & \text { Outlook } & 116\end{array}$

$\begin{array}{lll}7.5 & \text { Experimental } & 116\end{array}$

$\begin{array}{lll}7.6 & \text { References } & 119\end{array}$

Chapter 8: Self-Assembly of Molecular Motors on HOPG via

Bis(urea)tapes 121

$\begin{array}{lll}8.1 & \text { Introduction } & 122\end{array}$

8.2 Molecular design of bis(urea)-substituted molecular motors 123

8.3 Results and Discussion 125

8.3.1 Self-assembly of bis(urea)-substituted molecular support tapes $\quad 125$

8.3.2 Bis(urea)-substituted molecular motor tapes 126

8.3.3 Co-assembly bis(urea)-substituted motors and support molecules 128

8.3.4 New molecular designs for the support molecules 129

8.3.5 Co-assembly with the new support molecules 131

8.4 Conclusion 132

8.5 Outlook 132

8.6 Experimental 134

8.7 References 138

Abbreviations and Acronyms $\quad 141$

Nederlandse Samenvatting 143

$\begin{array}{ll}\text { Populair-Wetenschappelijke Samenvatting } & 145\end{array}$

$\begin{array}{ll}\text { Acknowledgements } & 149\end{array}$ 
Table of Contents 